\title{
Untersuchung der ergonomischen Eigenschaften von handbetätigten Mechanismen mittels Ebenen Force Feedback Interface (EFFI)
}

\author{
Bilgehan Demirkale ${ }^{1}$, Philipp Wabnitz ${ }^{2}$, Carsten Teichgräber ${ }^{3}$, Maik Berger ${ }^{4}$ \\ ${ }^{1}$ bilgehan.demirkale@s2013.tu-chemnitz.de \\ 2 philipp.wabnitz@mb.tu-chemnitz.de \\ ${ }^{2}$ carsten.teichgraeber@mb.tu-chemnitz.de \\ 2 maik.berger@mb.tu-chemnitz.de \\ 1,2,3,4 Professur Montage- und Handhabungstechnik, \\ Technische Universität Chemnitz, Deutschland
}

DOI: 10.14464/awic.v3i0.258

\begin{abstract}
Bei der Entwicklung handbetätigter Produkte mit ebenen Mechanismen spielen haptische Eigenschaften eine wichtige Rolle für die Kaufentscheidung des Kunden und sollten demnach so früh wie möglich in den Produktentwicklungsprozess einbezogen werden. Da CAD- und MKS-Modelle für die Simulation dieser nur bedingt geeignet sind, werden Prototypen hergestellt, welche hohe Kosten verursachen und bei Variantentests einen großen Zeitaufwand bedeuten. Deshalb kommen sogenannte haptische Feedbacksysteme als Werkzeug der virtuellen Produktentwicklung zum Einsatz. Hiermit lassen sich die ergonomischen Eigenschaften für verschiedene Modellvarianten frühzeitig ermitteln und mittels Nutzerstudien vergleichen. Das an der Professur entwickelte Ebene Force Feedback Interface (EFFI) wurde auf seine Eignung in dieser Hinsicht untersucht. Es kann gezeigt werden, dass die zuvor berechneten Bahnkurven vom Interface dargestellt werden können. Des Weiteren werden die haptischen Eigenschaften des Modells erfahrbar, wenn Nutzerkräfte am Griff angebracht werden. EFFI bietet somit das Potential ergonomische Studien bereits in einer frühen Entwicklungsphase durchführen zu können.
\end{abstract}

Keywords: Haptisches Feedback, virtuelle Produktentwicklung, Entwicklung ebener Mechanismen, Ebenes Force Feedback Interface (EFFI) 


\section{EINLEITUNG}

Im täglichen Leben haben Menschen vielfach Kontakt mit handbetätigten Produkten, wie bspw. Küchenschränken, Schubladen, Klappbetten o. Ä. in denen ebene Mechanismen zur Bewegungserzeugung integriert sind. Während der Interaktion mit diesen Produkten kann der Nutzer direkt den Komfort der Bedienung bzw. die ergonomischen und haptischen Eigenschaften dieses Produkts bewerten. Dabei stellen die erhaltenen haptischen Informationen in der Regel den ersten Anhaltspunkt zur Beurteilung der Produkteigenschaften und Qualität dar und haben einen Einfluss auf die Kaufentscheidung der Kunden (Klatzky, 2012).

Daher sollte zur frühzeitigen Beantwortung grundlegender bedientechnischer Fragestellungen noch während des Konstruktionsprozesses entweder auf reale oder virtuelle Prototypen zurückgegriffen werden. Der Aufbau realer Prototypen ist im Vergleich zu virtuellen Prototypen zeit- und kostenaufwendig. Unter diesen Umständen ist die Durchführung der Simulationen mit virtuellen Prototypen zwar der beste Weg, aber zur Untersuchung der taktilen Eigenschaften eines Produkts müssen realistische Abbildungen des physikalischen Verhaltens des Modells und der Interaktion zwischen dem Nutzer und dem Prototyp unbedingt integriert werden. Diese Anforderungen können nur mit der Verwendung der sog. haptischen Feedback Systeme (HFS) erfüllt werden, die die bidirektionale Informationsübertragung ermöglichen. Heute werden haptische Feedback-Technologien in verschiedenen Produkten und Bereichen, wie in Smartphones (Ryu, 2010), medizinischen Anwendungen (Kühnapfel, 2000), zur Ausbildung (Butnaru, 2011) oder für die Rehabilitation (Girone, 1999) erfolgreich eingesetzt. Daneben gewinnt die Verwendung der HFS in der virtuellen Produktentwicklung verstärkt an Bedeutung (Ferrise, 2013) (Shin, 2012). Ziel des Beitrages ist die Integration des an der Professur Montage- und Handhabungstechnik entwickelten Versuchsstandes EFFI in frühe Phasen des Entwicklungsprozesses von Mechanismen, um ergonomisch verbesserte und optimierte handbetätigte Produkte zu konstruieren.

Im Kapitel 2 werden Entwurfskriterien für das entwickelten HFS aufgeführt und der Versuchsstand wird kurz vorgestellt. Im folgenden Kapitel wird die Integration von EFFI in die Mechanismenentwicklung dargestellt. In Kapitel 4 werden die Ergebnisse von durchgeführten Variantentests eines Anwendungsbeispiels aufgezeigt, während das letzte Kapitel die daraus gewonnenen Erkenntnisse zusammenfasst.

\section{ENTWURFSKRITERIEN}

Abhängig vom Anwendungsbereich und Einsatzzweck wird eine Vielzahl von haptischen Geräten hergestellt. Viele handelsübliche Haptikgeräte besitzen verhältnismäßig kleine Arbeitsräume und sind für hochdynamische Anwendungen ungeeignet. Trotz der gegebenen Vielfalt an haptischen Displays existieren nur wenige kommerzielle Lösungen zur haptischen Simulation von großen Arbeitsräumen in Verbindung mit hohen Kräften (s. Abb.1). 
Im Vergleich zu räumlichen Mechanismen finden ebene Getriebe im Maschinenbau häufiger Verwendung, da sie einfacher $z u$ berechnen und aufzubauen sind. Auch viele der handbetätigen Bewegungseinheiten in unserem Alltag, wie z.B. Küchenschränke, Heckklappen und Schlafcouches, werden durch ebene Mechanismen geführt. Die zu deren Betätigung notwendigen menschlichen Bewegungen, wie Drücken, Ziehen, Heben oder Abstellen, sind vereinfacht ebenfalls als ebene Bewegungen zu betrachten. Demnach ist für den genannten Aufgabenbereich ein haptisches Gerät mit dem Freiheitsgrad F=2 auszulegen.

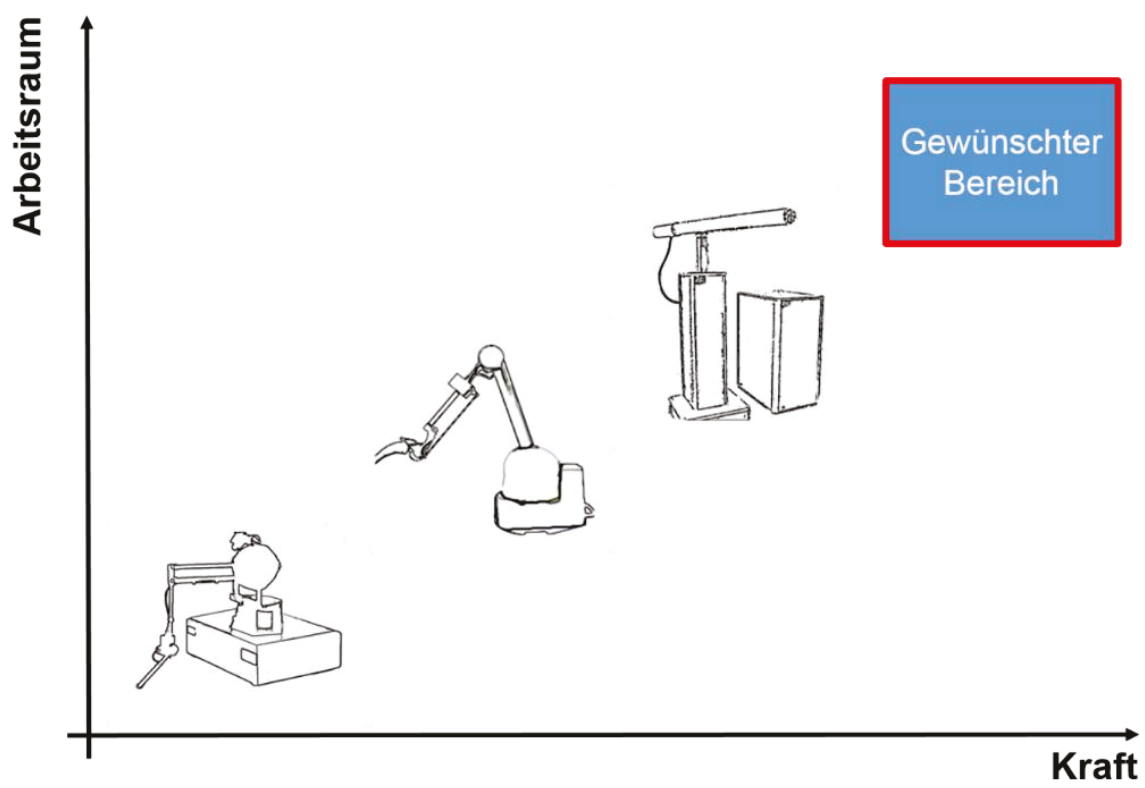

Abbildung 1 Einige haptische Geräte und der gewünschte Bereich im Vergleich

Quelle: eigene Darstellung

Ein wichtiges Entwurfskriterium zur Realisierung dieser Zielstellung ist die Komplexität des Systems. Das haptische Gerät soll sowohl hardwaretechnisch als auch softwaretechnisch möglichst einfach aufgebaut sein. Im Gegensatz zu anderen bekannten Systemen soll das Gerät aus der minimal notwendigen Anzahl an Gliedern und Gelenken aufgebaut sein. Die erhofften Vorteile sind zum einen die genaue Quantifizierbarkeit von Störgrößen, wie Toleranzund Reibungseinflüsse, Lagerspiel oder Übertragungseigenschaften, und zum anderen eine einfache Regelung mit klar beschriebenen Systemeigenschaften.

Die Software in Verbindung mit der Programmiersprache soll dem Konstrukteur eine anwenderfreundliche Programmieroberfläche bieten. Weiterhin sollten Parameterstudien und Simulationen durch eine unkomplizierte Parametervariation und kurze Rechenzeiten unterstützt werden. Variationsmöglichkeiten zur Bearbeitung verschiedener Aufgaben in realitätsnahen Abmessungen sind durch die Konfigurierbarkeit des Geräts zu gewährleisten. 
Daher bestand die Aufgabe, ein konzeptionell einfaches Ebenes Force Feedback Interface „EFFl“ zu entwickeln, welches große Arbeitsräume in Verbindung mit hohen Kräften realisieren kann (s. Abb. 2). EFFI ist ein admittanzgeregeltes haptisches Interface zur Darstellung der haptischen Eigenschaften ebener Mechanismen. Der Aufbau als planarer Roboter nach dem RPRPR-Schema ermöglicht es, Kräfte im Bereich von 0 bis $500 \mathrm{~N}$ in einem konfigurierbaren Arbeitsraum von 0,1 bis 1,2 $\mathrm{m}^{2}$ darzustellen. Eine ausführliche Beschreibung des Konzepts und der verwendeten Komponenten findet sich in (Demirkale, 2015) (Demirkale, 2017).
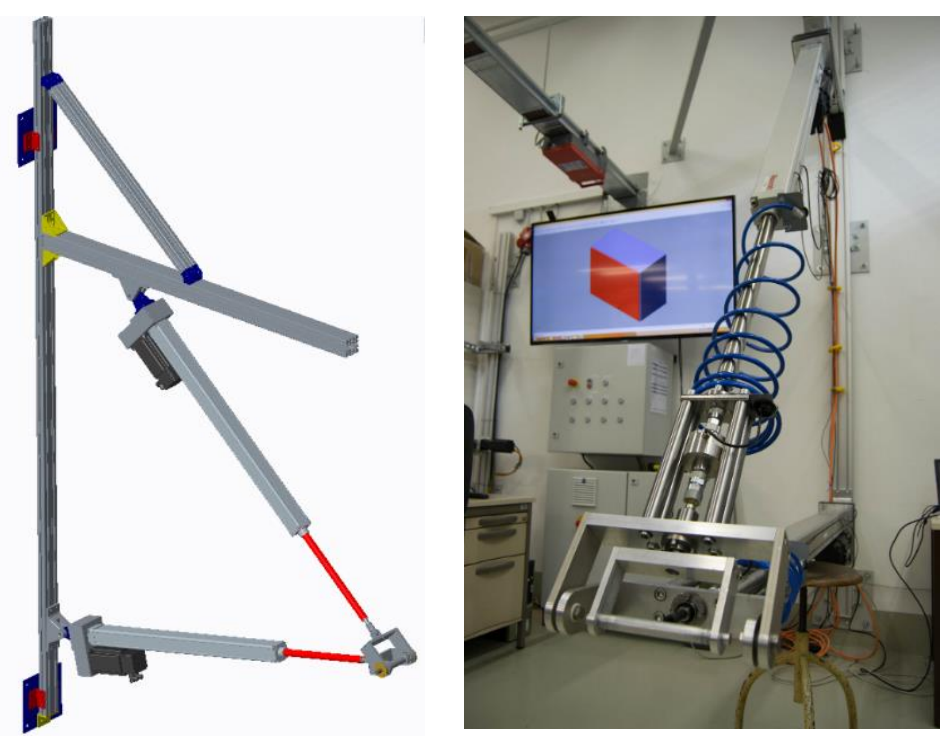

Abbildung 2: EFFI Versuchsstand. 3D-Modell von der Seite (links), Aufbau frontal (rechts) Quelle: $\quad$ eigene Darstellung

\section{INTEGRATION VON EFFI IN DIE MECHANISMENENTWICKLUNG}

In der klassischen Getriebesynthese werden zur Entwicklung eines Mechanismus die in Abb. 3-links dargestellten Prozessschritte betrachtet, die bis zum marktreifen Produkt in der Regel mehrfach durchlaufen werden müssen. Hierzu kann das haptische Feedback System (HFS) Abb.3-rechts in den gesamten Entwicklungsprozess des handbetätigten Mechanismus integriert werden. 


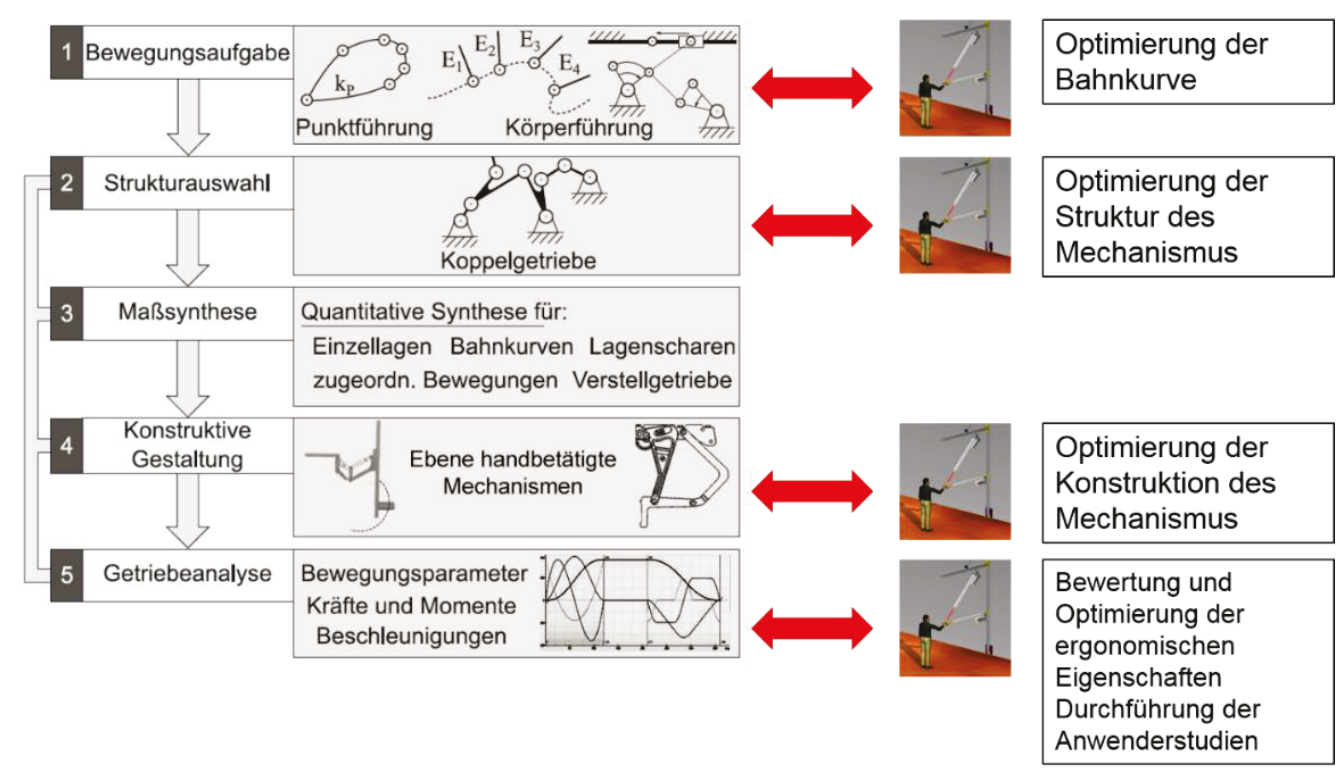

Abbildung 3: Klassisches Getriebesyntheseverfahren (links) und Einsatz des haptischen Feedback Systems im Getriebesyntheseverfahren (rechts)

Quelle: $\quad$ eigene Darstellung

Das haptische Feedback System kann sowohl als ein Unterstützungstool bei der Entwicklung für den Konstrukteur, als auch als ein virtueller Prototyp für Anwenderstudien verwendet werden. In dem ersten Schritt kann der Designer den Arbeitsraum gemäß der Randbedingungen einstellen und die Bahnkurve hinsichtlich der ergonomischen Parameter, wie z.B. Gelenkwinkel, Abstand zwischen der unteren und oberen Totlage, untersuchen und optimieren. Dabei kann er auch für zukünftige Anwenderstudien verschiedene Bewegungsmodellvarianten zum Testen bereitstellen. Nach der Erstellung der Struktur sind Getriebelängen und strukturbedingt entstehende Lasten mit Hilfe des haptischen Feedback Systems zu verbessern. Bei der konstruktiven Gestaltung haben vorkommende Änderungen wie z.B. Materialauswahl, verwendete kraftunterstützende Komfortelemente, konstruktiv bedingte entstehende Reibungen, einen großen Einfluss auf das ergonomische Verhalten des Mechanismus. Durch die Verwendung des HFS können diese Änderungen schnell getestet und verbessert werden. In dem letzten Schritt der Entwicklung können entwickelte Mechanismen hinsichtlich ihrer ergonomischen Eigenschaften bewertet und im Anschluss Anwenderstudien durchgeführt werden. Nach Auswertung des Benutzerfeedbacks kann der Designer aus den verschiedenen Varianten das ergonomisch optimale Produkt auswählen.

Die Implementierung der Simulation erfolgt nach einem festgelegten Prozessablauf (s. Abb.4). Das zu simulierende Produkt wird zunächst als CAD-Modell erstellt. Die Bahnkurve kann daraufhin in der CAD-Umgebung bestimmt und in diskrete Punkte zerlegt werden. Diese Informationen werden anschließend in der Steuerung hinterlegt. Ein vom CAD-Modell abgeleitetes Simscape MKS-Modell wird in Matlab/Simulink entsprechend der Simulationsbedingungen wie bspw. Masse- und Reibungsparameter oder eingesetzter 
Komfortelemente wie Federn und Dämpfer angepasst und für die Steuerung kompiliert. Anschließend ist das haptische Interface bereit für das haptische Rendering.

\begin{tabular}{|c|c|c|c|c|c|c|}
\hline Realer Mechanismus & CAD Modell & Ermittlung der Bahnkurve & MKS-Modell & Kompilierung & Haptische Simulation \\
\hline \hline & & & & \\
\hline
\end{tabular}

Abbildung 4: Prozessschritte der Simulation

Quelle: $\quad$ vgl. Demirkale (2017)

\section{ANWENDUNG AN EINEM FALLBEISPIEL}

Ein Küchenschrankmodell stellt ein repräsentatives Beispiel für die Verifikation und Validierung des im Kapitel 3 aufgezeigten Simulationsablaufs dar. Zur Simulation wurden verschiedene Modellvarianten mit und ohne eingesetzter Komfortelemente untersucht (s. Abb.5).

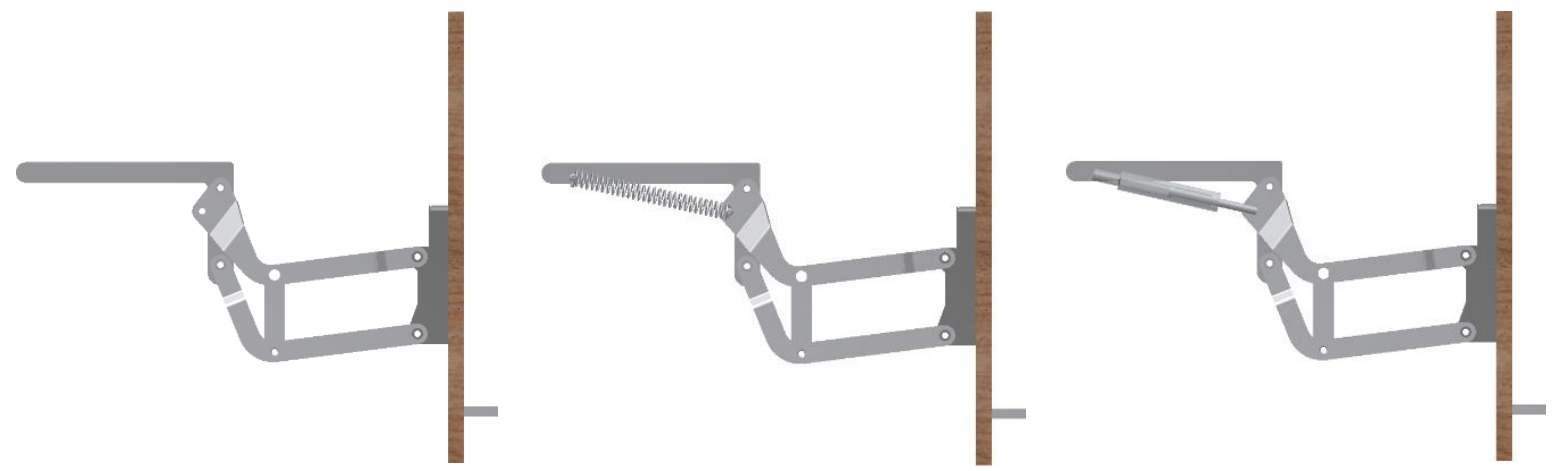

Abbildung 5: Varianten des Küchenschrank-Modells. Ohne Komfortelemente (links), mit Feder (mittig), mit Dämpfer (rechts)

Quelle: $\quad$ eigene Darstellung

Jede Modellvariante wurde am Versuchsstand ausgeführt und hinsichtlich ihrer dynamischen Eigenschaften untersucht. Hierbei wurde der Griff bis zum oberen Anschlagspunkt angehoben und anschließend fallengelassen. Das Führungsverhalten des Mechanismus konnte währenddessen beobachtet und durch den Bediener bewertet werden (s. Abb.6t). Da sich Versuchstand noch im Aufbau befindet, wurden die Versuche durch die Autoren des Beitrags durchgeführt. Nutzerstudien sind geplant, nachdem die Simulationsergebnisse an weiteren Modellen verifiziert werden konnten. 

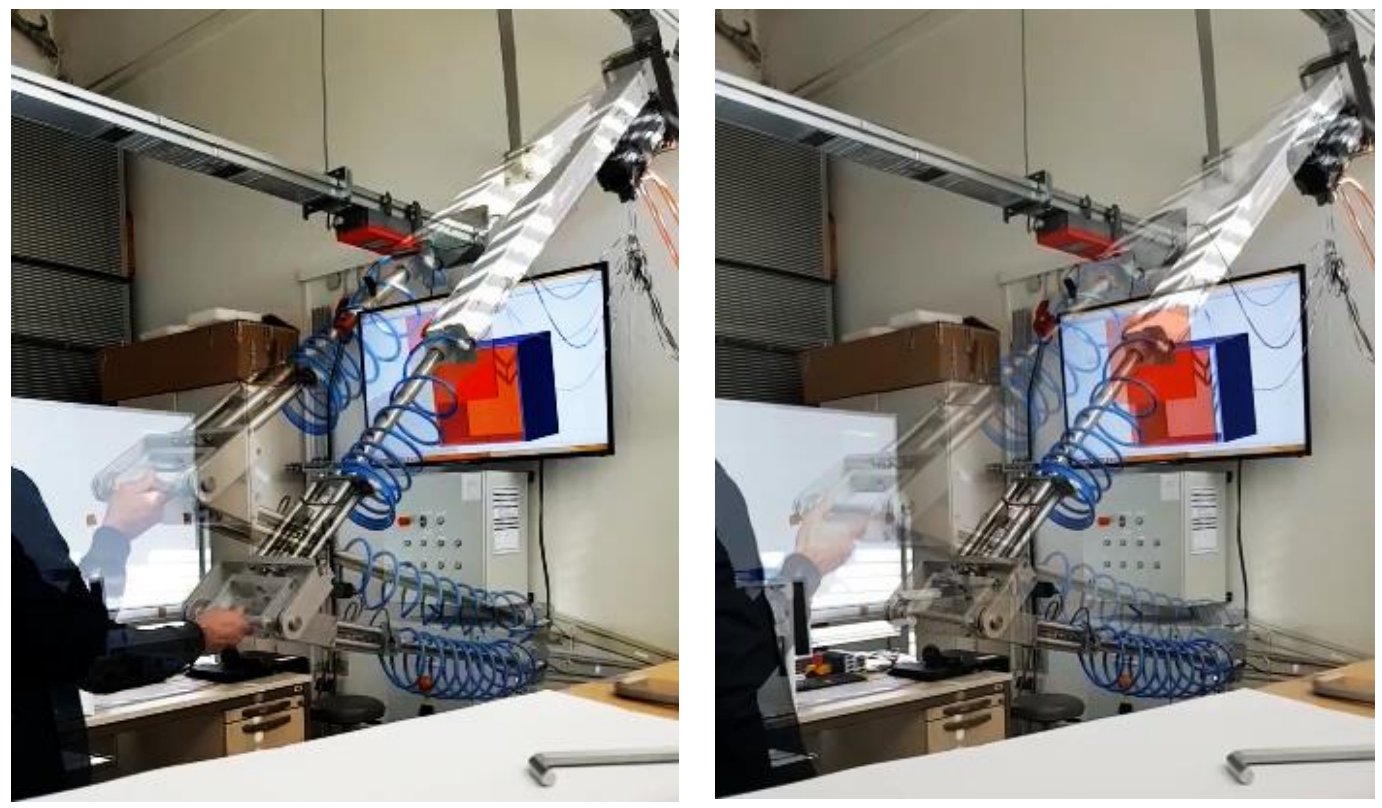

Abbildung 6: Untersuchungen am Versuchsstand. Heben (links), Fallenlassen des Griffs (rechts) Quelle: eigene Darstellung

Die vom haptischen Interface dargestellten Trajektorien des Griffs sind deckungsgleich mit der zuvor berechneten Bahnkurve des Küchenschranks (s. Abb.7). Zwischenwerte, welche aufgrund der vorgenommenen Diskretisierung nötig werden, wurden während der Simulation durch lineare Interpolation erzeugt. Sehr kleine Abweichungen ergaben sich lediglich in den Bereichen der Anschläge aufgrund der derzeitigen Leistungsbeschränkungen beim Rendering von Kontaktdefinitionen zwischen der Tür und dem Gestell.

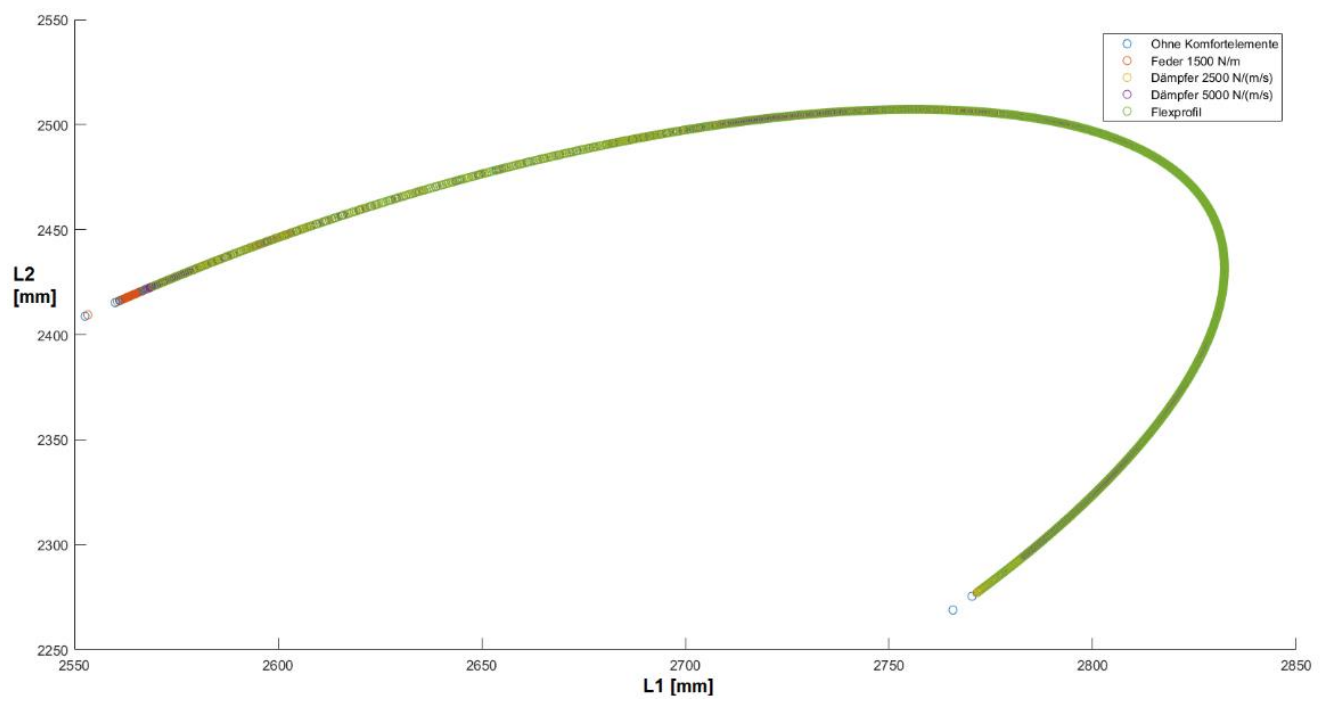

Abbildung 7: Vergleich der berechneten mit der durch EFFI realisierten Bahnkurven verschiedener Modellvarianten

Quelle:

eigene Darstellung 
Die Auswertung kinematischer Parameter erfolgte über die Aufzeichnung der Nutzerkraft, sowie der Griffposition und -geschwindigkeiten während der Simulation.
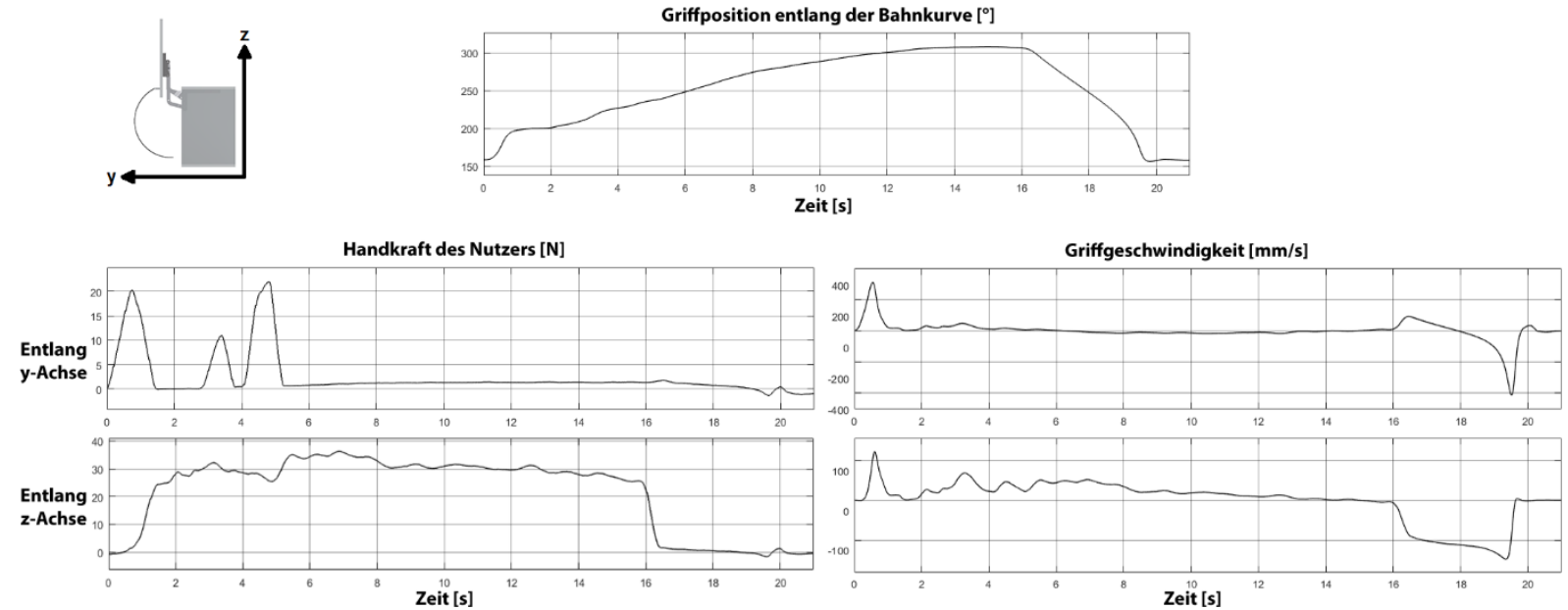

Abbildung 8: Ermittelte kinematische Parameter für die Bewertung des Komforts

Quelle: $\quad$ eigene Darstellung

Dies ermöglicht einen Vergleich mit gewonnenen Daten aus subjektiven oder objektiven Belastungsbewertungsverfahren, welche parallel durchgeführt werden können. Beispielhaft genannt sei hier der Einsatz der RPE-Skala nach Borg und physiologische Verfahren wie EMG-Messung an den Extremitäten (Mühlstedt, 2016).

Aus dem generierten Output lässt sich Folgendes ableiten (s. Abb.8):

1. Die gemessene Nutzerkraft entlang der Bahnkurve zeigt, wie viel Kraft der Nutzer an jeder Stelle der Führungsbewegung aufbringen muss, um die Tür aufzumachen bzw. zu schließen. Der Vergleich der Nutzerkraft entlang der Bahnkurve mit der subjektiven Auswertung des Nutzers hilft bei der Parametrierung des subjektiven Bedienkomforts. Außerdem hilft dieses Ergebnis in Kombination mit der beobachteten Körperhaltung bzw. den Gelenkwinkeln während der Bedienung bei der ergonomischen Evaluation.

2. Der Vergleich der Griffgeschwindigkeit mit den von EFFI ermittelten Daten zeigt, ob der Versuchsstand in der Lage ist, die in der MKS generierten, kinematischen Parameter zu realisieren.

3. Die subjektive Bewertung der Modellvarianten mit Hilfe einer einfachen Borg-Skala (RPE 6-20) erbrachte folgende Nutzerbewertungen:

- Das Modell mit Feder lässt sich nur schwer bewegen und wird als unangenehm empfunden (Wert: 17, „sehr anstrengend“)

- Das Modell mit Dämpfer wird als angenehm empfunden (Wert: 11, „leicht“)

- Das Modell ohne Komfortelemente wird als angenehm empfunden, jedoch ist das Heben der Tür schwer (Wert: 13, „etwas anstrengend“) 
Es ist geplant die gemessenen Parameter mit dem realen Verhalten des Küchenschranks unter Verwendung der simulierten Komfortelemente zu vergleichen und die Simulationsergebnisse somit zu verifizieren.

\section{ZUSAMMENFASSUNG}

Im Beitrag wurde die Integration von dem an der Professur Montage- und Handhabungstechnik entwickelten Ebenen Force Feedback Interface in den Entwicklungsprozess von Mechanismen aufgezeigt und hinsichtlich der Eignung zur Untersuchung ergonomischer Eigenschaften von ebenen Mechanismen getestet. Dieses weist im Gegensatz zu anderen haptischen Interfaces einen großen und konfigurierbaren Arbeitsraum auf und ermöglicht die Darstellung von hohen Kräften, welche bei der Benutzung von handbetätigten Produkten auftreten können.

Zu diesem Zweck wurden unterschiedliche Modellvarianten eines Küchenschranks erstellt, welche aufgrund des Einsatzes von Komfortelementen wie Federn und Dämpfer verschiedene ergonomische Eigenschaften aufwiesen. Diese virtuellen Prototypen konnten anschließend am Versuchsstand simuliert und hinsichtlich haptisch relevanter Parameter verglichen werden.

Es konnte gezeigt werden, dass das EFFI-System im Stande ist, Mehrkörpersimulationen von ebenen Getrieben auf Grundlage von CAD-Daten auszuführen. Es ermöglicht haptische Interaktionen an virtuellen Prototypen und erfült somit die Voraussetzung, Produkte nach ergonomischen Gesichtspunkten bereits in frühen Entwicklungsphasen zu evaluieren.

Aufgrund der Tatsache, dass sich das Interface noch im Aufbau befindet, ist mit einer weiteren Steigerung der Abbildungsgenauigkeit haptischer Eigenschaften zu rechnen. Nach Verifikation der Simulationsergebnisse an weiteren Modellen ist im nächsten Schritt eine Gegenüberstellung von subjektiven Komfortempfindungen und messtechnisch erfassten physiologischen Belastungsgrößen mit Hilfe von Probandentests geplant.

\section{LITERATURVERZEICHNIS}

Butnaru, T. (2011): et al. An approach for teaching mechanisms using haptic systems. In: The 6th Inter-national Conference on Virtual Learning ICVL. S. 30-36.

Demirkale, B.; Teichgräber C.; Berger. M. (2017): Haptisch-generierte nichtlineare Führungsbewegungen mit konfigurierbarem Ebenen Force Feedback Interface (EFFI). Fachtagung Mechatronik 2017: Dresden (09.03.-10.03.2017). (T. Bertram, B. Corves, \& K. Janschek, Hrsg.). Dresden: Technische Universität.

Demirkale, B.; Teichgräber. C.; Berger. M. (2015). Concepts and Implementations of a Haptic Simulation Method for Transfer and Guiding Mechanisms. TrC-IFToMM Symposium on Theory of Machines and Mechanisms, Izmir, Turkey, June 14-17, 2015

Ferrise, F., et al. (2013): Re-engineering of the Haptic Feedback of a Dishwasher Door. Computer-Aided Design and Applications, 10. Jg., Nr. 6, S. 995-1006. 
Girone, M.; Burdea, G.; Bouzit, M. (1999): The Rutgers ankle orthopedic rehabilitation interface. Proc. ASME Dyn. Syst. Control Div, 67. Jg., S. 305-312.

Klatzky, R. L.; Peck, J. (2012): Please touch - Object properties that invite touch. IEEE Transactions on Haptics, 5. Jg., Nr. 2, S. 139-147.

Kühnapfel, U.; Cakmak, H. K.; Maaß, H. (2000): Endoscopic surgery training using virtual reality and deformable tissue simulation. Computers \& graphics, 24. Jg., Nr. 5, S. 671682.

Mühlstedt J. (2016) Grundlagen virtueller Ergonomie. In: Bullinger-Hoffmann A., Mühlstedt J. (eds) Homo Sapiens Digitalis - Virtuelle Ergonomie und digitale Menschmodelle. Springer Vieweg, Berlin, Heidelberg

Ryu, J., et al. (2010): Psychophysical model for vibrotactile rendering in mobile devices. Presence: Teleoperators and Virtual Environments, 19. Jg., Nr. 4, S. 364-387.

Shin, S., et al. (2012): Haptic simulation of refrigerator door. IEEE Haptics Symposium (HAPTICS). IEEE, 2012. S. 147-154. 